\title{
Neural Activity in Human Primary Motor Cortex Areas 4a and 4p Is Modulated Differentially by Attention to Action
}

\author{
F. BINKOFSKI, ${ }^{1}$ G. R. FINK, ${ }^{2,3}$ S. GEYER, ${ }^{4}$ G. BUCCINO,${ }^{5}$ O. GRUBER,${ }^{6}$ N. J. SHAH ${ }^{3}$ J. G. TAYLOR, ${ }^{7}$ \\ R. J. SEITZ, ${ }^{1}$ K. ZILLES, ${ }^{3,4}$ AND H.-J. FREUND ${ }^{1}$ \\ ${ }^{1}$ Department of Neurology, University Hospital Düsseldorf, 40225 Düsseldorf; ${ }^{2}$ Department of Neurology, RWTH Aachen, \\ 52072 Aachen; ${ }^{3}$ Institute of Medicine, Research Center Jülich, 52425 Jülich; ${ }^{4}$ Department of Anatomy and C. \& O. Vogt \\ Brain Research Institute, Heinrich-Heine-University, 40225 Düsseldorf, Germany; ${ }^{5}$ Institute of Human Physiology, \\ University of Parma, 43100 Parma, Italy; ${ }^{6}$ Max-Planck-Institute for Cognitive Neuroscience, 04103 Leipzig, Germany; and \\ ${ }^{7}$ Department of Mathematics, Kings College, London WC2R 2LS, United Kingdom
}

Received 16 November 2001; accepted in final form 7 March 2002

Binkofski, F., G. R. Fink, S. Geyer, G. Buccino, O. Gruber, N. J. Shah, J. G. Taylor, R. J. Seitz, K. Zilles, and H.-J. Freund. Neural activity in human primary motor cortex areas $4 \mathrm{a}$ and $4 \mathrm{p}$ is modulated differentially by attention to action. J Neurophysiol 88: 514-519, 2002; 10.1152/jn.00947.2001. The mechanisms underlying attention to action are poorly understood. Although distracted by something else, we often maintain the accuracy of a movement, which suggests that differential neural mechanisms for the control of attended and nonattended action exist. Using functional magnetic resonance imaging (fMRI) in normal volunteers and probabilistic cytoarchitectonic maps, we observed that neural activity in subarea $4 p$ (posterior) within the primary motor cortex was modulated by attention to action, while neural activity in subarea $4 \mathrm{a}$ (anterior) was not. The data provide the direct evidence for differential neural mechanisms during attended and unattended action in human primary motor cortex.

\section{N T R O D U C T I O N}

Accurate performance of an action may require attention to many aspects thereof during its execution. This is most evident during the acquisition of a new motor skill. Many of our actions, however, have become automatic, e.g., walking or cycling, and we do not need to pay attention to them while they are performed. Also, in our every day life we often perform two or more actions in parallel while focusing our attention on only one of them. The following question then arises: how do we manage to maintain a sufficient level of control for such less or unattended actions?

It is known that prefrontal, anterior cingulate, and parietal cortices are engaged during controlled motor performance and that their degree of activation decreases the more a task becomes automatic (Grafton et al. 1995; Passingham 1996). By contrast, orienting gaze (and thus attention) toward an action may lead to a general increase in neural activity in several motor relevant areas including the primary motor cortex, as suggested by a recent functional magnetic resonance imaging (fMRI) study (Baker et al. 1999). In our current study we used fMRI in normal volunteers to investigate the neural mechanisms associated with a stereotyped performance of a move-

Address for reprint requests: F. Binkofski, Dept. of Neurology, University Hospital Lübeck, Ratzeburger Allee 160, 23538 Lübeck, Germany (E-mail: binkofski.f@neuro.mu-luebeck.de). ment while gradually changing the amount of attention to this action to identify structures differentially engaged in the control of attended and nonattended action. Accordingly, we chose a dual visual and motor task in which subjects were asked to perform 1) a stereotyped right index finger movement that required no learning and 2) a visual distractor task. The latter was introduced to allow us to modulate subjects' levels of attention to motor task performance without interfering with movement type, amplitude, and frequency (Fig. 1). Kinematic recordings confirmed that mean frequency and mean amplitude of the forefinger movements did not differ between the three experimental conditions.

A preliminary account was published in abstract form (Binkofski et al. 1998).

\section{METHODS}

\section{Subjects}

Six healthy, right-handed volunteers, 25-35 yr of age (5 males and 1 female) participated in this study after providing informed consent. Handedness was assessed by the Oldfield inventory (Oldfield 1971). The study was approved by the local ethic committee of the HeinrichHeine-University Düsseldorf.

\section{Experimental procedure}

Subjects were asked to move their right index finger back and forth in the form of a well-shaped "U" (Fig. 1A) at a constant amplitude and a frequency of $1.5 \mathrm{~Hz}$ (paced by a metronome to keep the movement constant throughout all experimental conditions), while they looked at a video display unit presented in the MR scans through a mirror. A red screen was presented in condition 1 with occasional short intermittent flashes of green light $(100 \mathrm{~ms})$ in conditions 2 and 3. Four to seven such flashes were presented in different time intervals in one scanning block (10 s). Prior to fMRI scanning subjects were trained to perform the four different tasks (3 experimental conditions plus baseline): 1) directed attention to the moving finger, while looking at the screen but not paying attention to it (condition 1); 2) directed attention to the screen and not counting the intermittent short flashes of green, while

\footnotetext{
The costs of publication of this article were defrayed in part by the payment of page charges. The article must therefore be hereby marked "advertisement" in accordance with 18 U.S.C. Section 1734 solely to indicate this fact.
} 
A

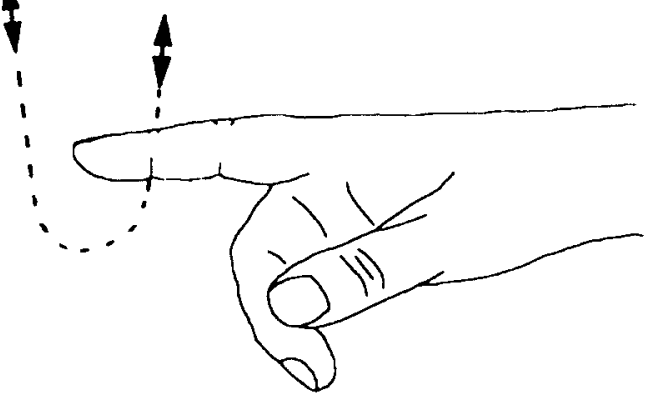

B

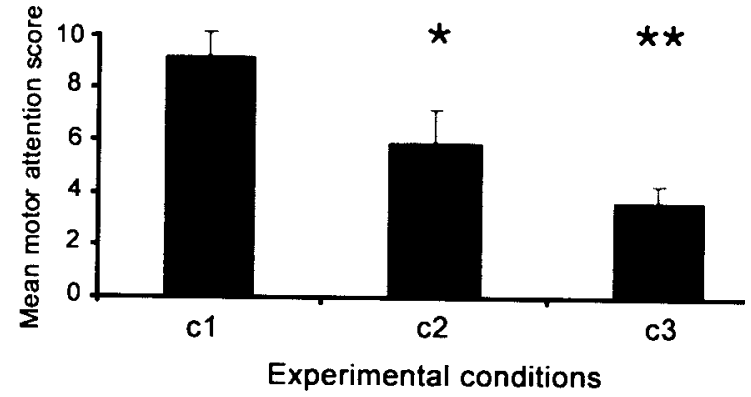

FIG. 1. Behavioral data. A: schematic drawing of a hand performing the stereotyped forefinger movements in the form of a well-shaped "U." $B$ : subjective mean levels of attention (and SDs) to the finger movements in all subjects in all 3 active conditions (C1, C2, and C3) as assessed by the analog scale. Note the significant statistical decrease in the level of attention to the finger movement between each condition as assessed by a 2 -tailed $t$-test $(* P<$ 0.05 ; ** $P<0.01)$.

performing the stereotyped finger movements (condition 2); 3) directed attention to the screen and counting the flashes of green, while performing the stereotyped finger movements (condition 3); and 4) directed attention to the screen and the flashes of green without any finger movement (baseline). As assessed in a control study, in the three different experimental conditions a sufficient level of graded attention to the motor task was achieved without affecting the performance of the task (see RESULTS). For control the subjects were asked to report the number of perceived flashes of green after each scanning session. The reported numbers of green flashes were always correct, thus providing evidence that subjects performed the task adequately. The hands were precluded from vision. Measurements were arranged in four blocks, each containing all four conditions, in randomized order, separated by a rest period (no movement, no visual stimulation). Each experimental condition, the baseline, and the rest period lasted for $50 \mathrm{~s}$.

After each fMRI measurement block, subjects 1) rated their level of attention to the finger movements during scanning using an analog scale from 10 (maximum attention to the finger movements) to 1 (no attention to the finger movements) and 2) reported their count of the number of green flashes during conditions 3 (high level distraction from the motor task) and 4 (baseline). The employment of the analog scale was practiced and tested prior to the scanning procedure. Right index finger movements were recorded with two-axis goniometers (Peny and Giles, Christchurch, UK) placed around the metacarpophalangeal joint. The signals from the goniometers were recorded with a multichannel CED System (Cambridge Electronics) and further analyzed with the Spike2 software package. The amplitudes of all movement periods were pooled for each condition, and mean values and SDs were calculated. The frequency distribution was assessed by means of power spectrum analysis. The values were then pooled for each condition, and also mean values and SDs were calculated.

\section{Scanning procedure and data analysis}

Brain activity was measured by fMRI using echo planar imaging (EPI) to exploit the blood oxygen level dependent (BOLD) effects. BOLD contrast image volumes were acquired at $1.5 \mathrm{~T}$ (Siemens VISION) with gradient-echo, echo-planar imaging (TR/TE $=5,000$ $\mathrm{ms} / 66 \mathrm{~ms}, \alpha=90^{\circ}$ ). Each volume comprised 30 contiguous 4-mm slices, with an in-plane resolution of $3 \times 3 \mathrm{~mm}$. Each subject underwent four consecutive imaging sessions comprising 320 such volumes. The first 10 volumes of each session were discarded to circumvent T1 saturation effects. For each subject separately, the EPI time-series images were realigned to the 20th image of each measurement, stereotactically normalized and smoothed with an isotropic Gaussian kernel of 8-10 mm FWHM resulting in an in-plane resolution of approximately $8 \mathrm{~mm}$ (Friston 1995; Friston et al. 1995, 1996). The entire imaging time series for each subject was used for a group analysis, representing 1,920 image volumes in total. Condition-specific effects were estimated using the "General Linear Model" and theory of Gaussian random fields as implemented in SPM97. A high-pass filter with a cutoff frequency of 0.19 cycles per min modeled and excluded low-frequency confounding effects in the time series. Adjusted voxel means for each condition and the adjusted error variance were generated. The differences between conditions were assessed by weighting the condition means with the appropriate contrast conditions. An additional conjunction analysis (Price and Friston 1997) was performed for all experimental conditions (conditions 1,2, and 3) relative to the baseline. This analysis reveals the areas that behave congruently irrespective of the given level of attention. The imaging data were also compared with the subjective attention scores. Multiple subjects and the replication of conditions were taken into account by using linear contrasts to test hypothesis of regionally specific condition effects. The statistical parametric map $\operatorname{SPM}\{Z\}$ for all comparisons was thresholded at a $Z$ value of $3.09(P=0.001$ uncorrected for multiple comparisons), and the resulting foci were characterized in terms of both spatial extent and peak height corrected for multiple comparisons at the 5\% level (Friston 1995; Friston et al. 1995, 1996).

\section{Comparison with the probabilistic maps}

Cytoarchitectonic mapping of areas $4 \mathrm{a}$ and $4 \mathrm{p}$ was performed in 10 postmortem human brains obtained at autopsy from subjects with no history of neurological or psychiatric diseases. All brains were obtained through the body donor program of the Department of Anatomy, University of Duesseldorf, Germany. The brains were suspended at the basilar artery and fixed for approximately 5 mo in $4 \%$ formaldehyde or Bodian's fixative. After fixation, T1 weighted MR scans [1.5 T Siemens Magnetron SP scanner, 3-D fast low angle shot (FLASH) sequence, flip angle $40^{\circ}$, TR $40 \mathrm{~ms}$, TE $5 \mathrm{~ms}$ ] were acquired for documentation of brain size and shape before histological processing. The brains were dehydrated in graded alcohols, embedded in paraffin, and sectioned coronally ( $20-\mu \mathrm{m}$ whole brain sections). Images of the paraffin blockface were obtained after each 60th section with a charge-coupled device (CCD) camera. Each 60th section was mounted on a gelatin-coated slide and stained for cell bodies with a Nissl-like method (Merker 1983).

Rectangular regions of interest (ROIs) covering the right and left precentral gyrus were defined in each cell-stained section. In each ROI, the areal fraction of darkly stained cell bodies (gray level index; GLI) was measured after adaptive thresholding (Schleicher and Zilles 1990) in square, adjoining fields (size $27 \times 27 \mu \mathrm{m}$ ). The resulting data matrix covering the entire ROI is the GLI image (Schleicher and Zilles 1990). Equidistant density profiles (297 $\mu \mathrm{m}$ wide, oriented orthogonally to the cortical layers and extending from the border between layers I and II to the border between layer VI and the white matter, spacing between adjacent profiles $297 \mu \mathrm{m}$ ) were extracted from each GLI image and standardized to a cortical depth of $100 \%$ by resampling the data with linear interpolation. To quantify each profile's shape, 10 numerical features based on the laminar neuronal 
densities (e.g., mean, skewness, kurtosis) were calculated for each profile and combined into one feature vector. A mean feature vector was calculated from a block of 10 adjacent profiles, and another mean vector from a neighboring block of 10 adjacent profiles. Differences between the mean feature vectors from two neighboring blocks of profiles were calculated as Mahalanobis distances $D^{2}$ (Mahalanobis et al. 1949). $D^{2}$ values were plotted as a function of the positions of the profile blocks relative to the cortex. The resulting distance function revealed maxima where the regions covered by profiles showed differences in their laminar patterns. Statistical significance was evaluated by a Hotelling's $T^{2}$ test. The positions of significant maxima were then compared with the cytoarchitectonic pattern (for numerical data see Geyer et al. 1996; for further technical details see Geyer et al. 1999; Schleicher et al. 1999, 2000).

Each mounted and cell-stained histological section was digitized with a CCD camera. The histological volume of the brain was then reconstructed in three dimensions (3-D) from the images of the paraffin blockface, the digitized histological sections, and the MR volume of the same brain with linear and nonlinear transformations (Schormann et al. 1996). With an interactive voxel-painting program the extent of areas $4 \mathrm{a}$ and $4 \mathrm{p}$ was transferred from the histological sections to the corresponding sections of the reconstructed volume. Thus a microstructurally defined representation of cytoarchitectonic areas $4 \mathrm{a}$ and $4 \mathrm{p}$ was obtained in the $3-\mathrm{D}$ reconstructed histological volume of each brain. Each histological volume (with the representations of areas $4 \mathrm{a}$ and $4 \mathrm{p}$ ) was then spatially normalized to the reference brain of a computerized atlas (which is oriented in the Talairach coordinate system) (see Roland et al. 1994) with an algorithm based on an extended principal axes theory and a fast automated multiresolution full-multigrid movement model (Schormann and Zilles 1998). The 10 normalized histological volumes were superimposed in the 3-D space of the reference brain. A population map was generated for each area that shows the degree of interindividual microstructural variability by exemplifying, for each voxel, how many brains have a representation of this cytoarchitectonic region.

With the same warping algorithm the high-resolution Montreal Neurological Institute template and the SPM maps were aligned to the reference brain. The functional activation foci were then superimposed with the $50 \%$ isocontour (i.e., representation in $\geq 5$ brains) of each area's population map. The percentage of overlap between the volumes of the activation foci and the volumes of the $50 \%$ isocontour of areas $4 \mathrm{a}$ and $4 \mathrm{p}$ was calculated.

\section{RE S ULTS}

\section{Morphological features of area $4 a$ and $4 p$}

The primary motor cortex (Brodmann's area 4) is located in the precentral gyrus. The caudal border of area 4 (toward the primary somatosensory cortex) lies in the depth of the central sulcus close to its fundus. The nonprimary motor cortex (Brodmann's area 6) rostrally abuts on area 4. Dorsomedially on the cortical convexity (toward the midline), the border between area 4 and 6 lies on the exposed cortical surface on the vertex of the precentral gyrus. Further ventrolaterally (toward the Sylvian fissure), it recedes in a caudal direction and eventually disappears in the depth of the central sulcus. Areas $4 \mathrm{a}$ and $4 \mathrm{p}$ are two parallel bands within area 4 (rostral band: $4 \mathrm{a}$, caudal band: $4 p$ ) running mediolaterally from the midline to the Sylvian fissure. Lower layer III pyramidal cells are small and loosely aggregated in area $4 \mathrm{p}$, larger and more densely packed in area $4 \mathrm{a}$, and even larger, more elongated, and sometimes arranged in several parallel rows like a phalanx in area 6 . There are no differences in size, packing density, or arrangement of giant pyramidal cells between areas $4 \mathrm{a}$ and $4 \mathrm{p}$ (Geyer et al. 1996).

\section{Behavioral data}

The kinematic recordings did not show any significant differences between the three experimental conditions regarding the mean frequency (condition 1: $1.4 \pm 0.07 \mathrm{~Hz}$, mean $\pm \mathrm{SD}$; condition 2: $1.44 \pm 0.11 \mathrm{~Hz}$; condition $3: 1.38 \pm 0.49 \mathrm{~Hz})$ and mean amplitude (condition 1: $4.9 \pm 0.58 \mathrm{~cm}$; condition 2: $5.1 \pm 0.6 \mathrm{~cm}$; condition 3: $5.0 \pm 0.49 \mathrm{~cm}$ ) of the U-shaped finger movements. The analog assessment of the subjective levels of attention to movement, however, showed that significantly different values were reached in each condition (condition 1: $9.24 \pm 0.07$; condition 2: $5.95 \pm 1.28$; condition 3: $3.71 \pm 0.49$; Fig. $1 B)$.

\section{Comparison between activation foci and the probabilistic maps}

The conjunction analysis (Price and Friston 1997) of all active conditions (conditions 1, 2, and 3; each contrasted with the baseline) revealed significant activation of the primary motor cortex (BA 4), the right cerebellum, and extrastriatal areas on both sides (Table $1 A$ ).

TABLE 1. Significant activation areas

\begin{tabular}{lcr}
\hline \hline \multicolumn{1}{c}{ Activated Area } & Stereotactic Coordinates & Z-Score \\
\hline \multicolumn{4}{c}{ A. Conjunction of active conditions versus control } \\
Area 4 & $-48,-8,56$ & 5,1 \\
Cerebellum & $12,-56,-16$ & 3,9 \\
Extrastriatal r & $20,-96,12$ & 5,3 \\
Extrastratla 1 & $-30,-92,8$ & 3,8
\end{tabular}

Extrastratla 1

B. Parametric changes in area 4 4a, not modulated $4 \mathrm{p}$, modulated
Prefrontal $r$

vPMC r

Superior parietal $\mathrm{r}$

Secondary sensory $r$

Intraparietal $\mathrm{r}$ Temporo-occipital

$\mathrm{r}$

\section{DLPC r}

vPMC 1

Posterior parietal $r$

Precuneus $r$

Fusiform gyrus $r$

Fusiform gyrus 1

Extrastriatal $r$

Extrastriatal 1

Primary visual 1

$$
-48,-8,56
$$$$
-36,-20,48
$$

5,1

5,3

C. Motor-directed attention

$\begin{array}{ll}20,60,1 & 3,2 \\ 56,16,8 & 3,3 \\ 36,-48,62 & 3,8 \\ 56,-28,24 & 4,3 \\ 28,-56,52 & 3,3 \\ 44,-60,16 & 3,2\end{array}$

D. Visually directed attention

$$
\begin{gathered}
44,40,28 \\
-56,8,40 \\
56,-52,44 \\
4,-56,48 \\
48,-86,-20 \\
-52,-56,-12 \\
32,-76,-16 \\
-16,-84,-12 \\
-8,-84,8
\end{gathered}
$$

Stereotactic coordinates in $\mathrm{mm}$ in Talairach apace (Talairach and Turnoux 1988). A: conjunction analysis of all active conditions in contrast to the control condition (condition $1+$ condition $2+$ condition $3>$ condition 4 ) showing the common activation areas related to right index finger movements. $B$ : parametric evaluation of neural activity in Area 4 that was modulated (area 4p: posterior) and not modulated (area 4a: anterior) by attention to finger movement. $C$ : motor-directed attention (condition $1>$ condition $2+$ condition 3 ). $D$ : visually directed attention (condition $2+$ condition $3>$ condition 1 ). Area 4, Brodman's Area 4; modulated, modulated by attention to action; not modulated, not modulated by attention to action; vPMC, ventral premotor cortex; DLPF, dorsolateral prefrontal cortex. 
A

\section{Focus Modulated by Attention}

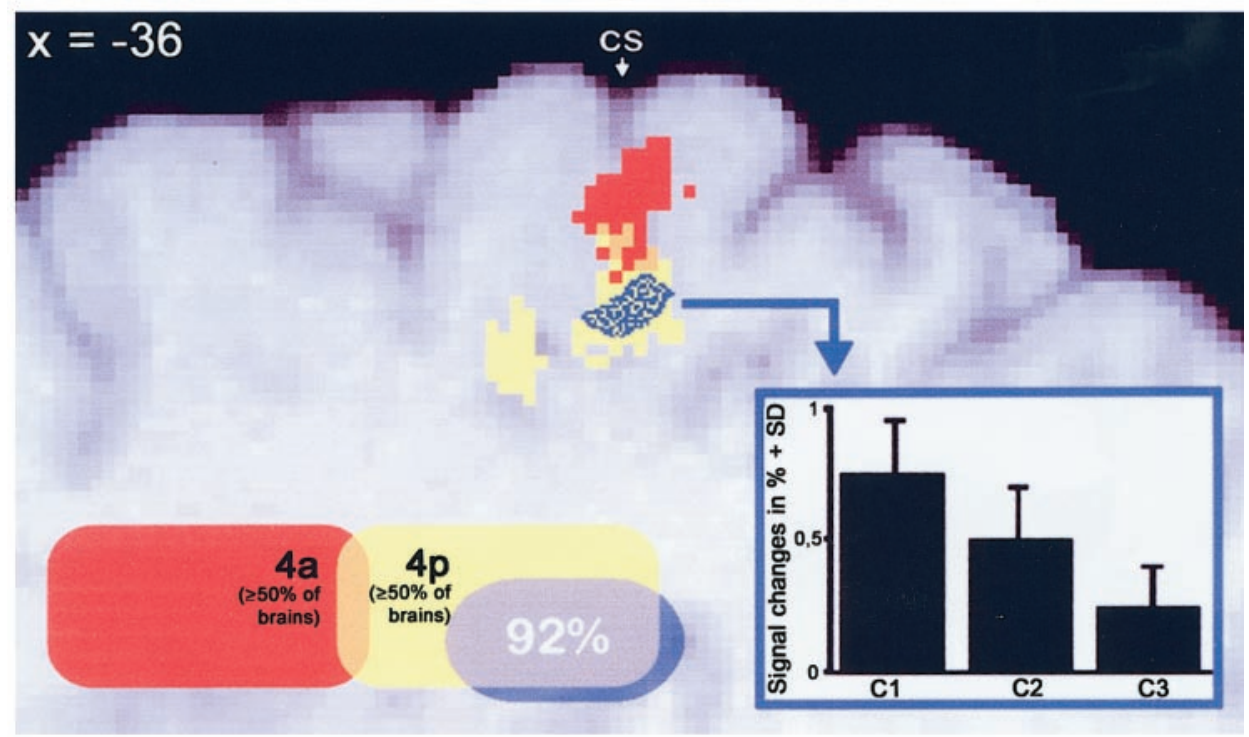

B

\section{Focus not Modulated by Attention}

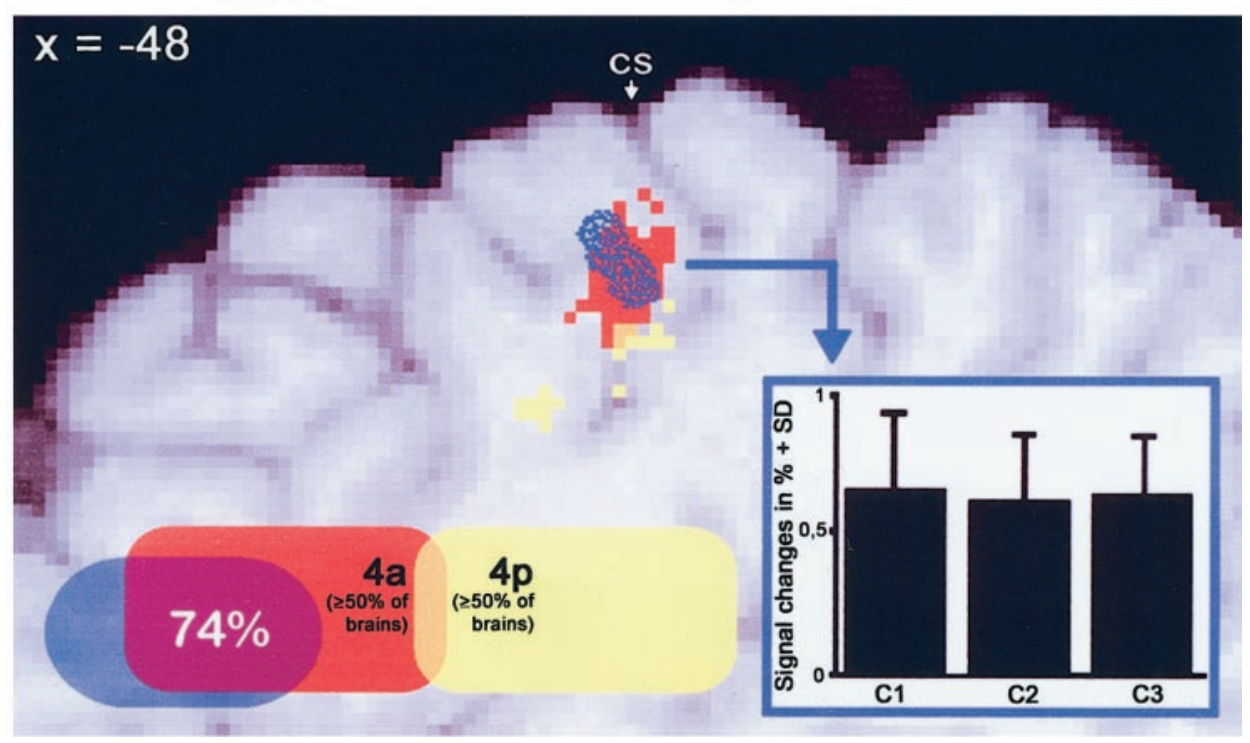

FIG. 2. Overlap of the group activation foci in the primary motor cortex with the probabilistic cytoarchitectonic maps of areas $4 \mathrm{a}$ (anterior) and $4 \mathrm{p}$ (posterior). The activated foci are in blue, the area $4 \mathrm{a}$ in red, and the area $4 \mathrm{p}$ in yellow. The different sagittal transsection planes through the central sulcus (CS) show the center of activity of the focus modulated by attention in the area $4 \mathrm{p}$ (top panel) and the center of activity of the focus not modulated by attention in the area $4 \mathrm{a}$ (bottom panel). The percentage of the overlap between the respective cytoarchitectonic region and the activated focus are illustrated in the bottom left corner. The percentage of the mean signal changes, and the SDs in the corresponding activated area are depicted in the bottom right corner. A: the focus modulated by the level of attention overlapped by $92 \%$ with the $50 \%$ isocontour of the area $4 \mathrm{p}$. Talairach coordinates $(x=-36, y=-20, z=48)$. B: the focus not modulated by attention overlapped by $74 \%$ with the $50 \%$-isocontour of the area 4a. Talairach coordinates $(x=-48, y=-8$, $z=56)$.

Parametric comparison of the signals with attention scores revealed that the BOLD signal in the depth of the central sulcus co-varied with the subjective levels of attention to action (Table 1B; Fig. 2A). By contrast, the neural activity in a more lateral part of the central sulcus did not show such a covariation (Table $1 B$; Fig. $2 B$ ). To assess whether these differential responses belonged to different subareas of primary motor cortex, the local maxima within these activation areas were co-registered with the probabilistic cytoarchitectonic population maps of areas $4 \mathrm{a}$ and $4 \mathrm{p}$ of the human primary motor cortex (Geyer et al. 1996). The focus modulated by attention overlapped by $92 \%$ with area $4 p$ but did not overlap at all with area $4 \mathrm{a}$ (Fig. 2A). By contrast, the focus that was not modulated by attention overlapped by $74 \%$ with area $4 a$, and extended into area 6 (Fig. $2 B$ ), but did not overlap with area $4 p$. The real world distance between the centers of gravity of the two foci was $19 \mathrm{~mm}$, and there was no overlap between two of them. The anatomical location of these two differentially modulated activation foci within area 4 and their relationship to the cytoarchitectonic probabilistic maps of areas $4 a$ and $4 p$ are shown in Fig. 2.

The categorical comparison between the experimental conditions with a high level of motor-directed attention (condition 1) and a high level of visual-directed attentions (condition 3) yielded the following differences. Motor-directed attention relative to visually directed attention (condition $1>$ condition $2+$ condition 3 ) revealed increased neural activity basically in a right parietal-prefrontal circuit (prefrontal cortex, ventral 
premotor cortex, superior parietal, secondary somatosensory area, intraparietal sulcus and temporo-occipital cortex; Table $1 C)$. By contrast, while visually directed attention relative to motor-directed attention (i.e., condition $2+$ condition $3>$ condition 1) revealed a bilateral circuit involving extrastriate and dorsolateral prefrontal cortex (right dorsolateral prefrontal cortex, left ventral premotor cortex, right posterior parietal cortex, right precuneus, bilateral fusiform gyrus, bilateral extrastriatal cortex and left primary visual cortex; Table $1 D$ ).

\section{I S C U S S I O N}

Our data shed light on the basic mechanisms underlying attention to action. The novel finding here was the observation that neural activity within human primary motor cortex is modulated differentially by attention. This novel finding parallels previous reports of neural activity in primary and secondary sensory cortices being modulated by attention in the visual, auditory, and somatosensory domains (Corbetta et al. 1993; Desimone and Duncan et al. 1995; Fink et al. 1996; Iriki et al. 1996; Shulman et al. 1997; Steinmetz et al. 2000; Woldorf et al. 1993) and extends current concepts of primary motor cortex function: attentional modulation of human primary motor cortex activity strongly questions the classical and simplistic view of the human primary motor cortex as a pure somatotopically organized executive motor structure (DennyBrown and Botterell 1948; Foerster 1936; Leyton and Sherrington 1917; Penfield and Rasmussen 1950).

On the basis of recent cytoarchitectonic data showing a subdivision of the primary motor cortex (Geyer et al. 1996), we observed that these distinct subregions within primary motor cortex show differential attentional modulation during motor performance. These differentially modulated foci were $19 \mathrm{~mm}$ apart from each other and could therefore be clearly separated given the spatial resolution of $8-10 \mathrm{~mm}$ of our images even when one also takes into account a spatial dispersion of the BOLD-response of 3-5 mm (Malonek and Grinvald 1996). There was an attention-modulated area within cytoarchitectonically defined area $4 p$ in the depth of the central sulcus and another area within the lateral part of the posterior bank of the precentral gyrus that was not modulated by attention. The latter area included cytoarchtectonically defined area $4 \mathrm{a}$ and extended into premotor area 6. Although our understanding of anatomical and functional parcellation within human primary motor cortex is only at its beginning, based on our data we hypothesize that these separate regions within human primary motor cortex may belong to different motor channels that allow for parallel processing of motor information with different attentional load in situations that necessitate simultaneously attended and unattended action. Interestingly, differential anatomical connections have also been demonstrated for these areas: area $4 \mathrm{p}$, which occupies the deep part of the posterior bank of the precentral gyrus, is primarily connected with the primary sensory cortex (Stepniewska et al. 1993). The modulation of primary sensory cortex by a motor task (Hsiao et al. 1993; Iriki et al. 1996) could thus help to explain the attentional modulation of area $4 \mathrm{p}$ in our current study. Area $4 \mathrm{a}$, which rostrally abuts on area $4 \mathrm{p}$ and lies more superficially toward the free surface, is connected to the premotor cortex (Stepniewska et al. 1993). These subareas of primary motor cortex have different thalamic connections in the owl monkeys
(Stepniewska et al. 1994); however, whether such connections also exist in the human brain remains to be investigated.

Geyer et al. (1996) have already provided some data suggesting a differential specialization of $4 \mathrm{a}$ and $4 \mathrm{p}$, when showing that a roughness discrimination task activated area $4 p$ relatively more than self-generated movements. Here we show the differential mode of neural activity in areas $4 \mathrm{a}$ and $4 \mathrm{p}$ according to the amount of attention directed toward the action. A parsimonious explanation for the observed modulation in area $4 \mathrm{p}$ in our study may then be that increased motor-directed attention may also include increased attention to sensory feedback, which in turn could have led to increased neural activity in area 4 p. Likewise, it seems possible, although speculative at present, that area $4 \mathrm{a}$ of primary motor cortex might be responsible for maintaining the execution of a motor program, irrespective of the amount of attention paid to it.

The demonstration of attentional modulation of primary motor cortex supports the cognitive role of human primary motor cortex in line with electrophysiological evidence obtained from animal experiments: nonhuman primate M1 neurons are capable of "holding in memory" movement direction, motor sequences, and the serial order of movements (Carpenter et al. 1999; Pellizer et al. 1995). Neurons within area 4 were also shown to be involved in mental rotation (Lurito et al. 1991). More recently, the existence of motor output independent higher-order representations of task objectives and constraints in M1 were suggested on the basis of single joint movement experiments in monkeys (Shen and Alexander 1997). Such monkey electrophysiological evidence is supplemented by magnetoencephalographical studies in humans, which implicate M1 in motor imagery and movement observation (Hari et al. 1998; Schnitzler et al. 1997). Thus our functional imaging results and previous data support the notion that human primary motor cortex function goes beyond simple motor output.

\section{REFERENCES}

BAKer JT, Donoghue JP, AND SANES JN. Gaze direction modulates finger movement activation patterns in human cerebral cortex. J Neurosci 19: 10044-10052, 1999.

Binkofski F, Buccino G, Taylor JG, Gruber O, Posse S, Shah NJ, Freund HJ, AND SEITZ RJ. Attention modulates motor cortex activation. An fMRI study (Abstract). Neuroimage 7: 84, 1998.

Carpenter AF, Georgopoulos AP, and Pellizzer G. Motor cortical encoding of serial order in a context-recall task. Science 283: 1752-1757, 1999.

Corbetta M, Miezin FM, Shulman GL, and Petersen SE. A PET study of visuospatial attention. J Neurosci 13: 1202-1226, 1993.

DENNY-BRown D AND BotTERELl EH. The motor functions of the agranular frontal cortex. Res Publ Assoc Res Nerv Ment Dis 27: 235-345, 1948.

DESIMONE R AND DunCAN J. Neural mechanisms of selective visual attention. Annu Rev Neurosci 18: 193-222, 1995.

Fink GR, Halligan PW, Marshall JC, Frith CD, Frackowiak RSJ, and DOLAN RJ. Where in the brain does visual attention select the forest and the trees? Nature 382: 626-628, 1996.

FoERSTER O. Motorische Felder und Bahnen. In: Handbuch der Neurologie, edited by Bumse H and Foerster O. Berlin: Springer, 1936, vol. 6, p. 1-37.

Fox P, Miezin FM, Allman JM, Van Essen DC, and Reichle ME. Retinotopic organization of human visual cortex mapped with positron-emission tomography. J Neurosci 7: 913-922, 1987.

FRISTON KJ. Statistical parametric maps in functional imaging, a general linear approach. Hum Brain Mapp 2: 189-210, 1995.

Friston KJ, Ashburner J, Frith CD, Poline J-B, Heather JD, And FrackOWIAK RSJ. Spatial registration and normalization of images. Hum Brain Mapp 2: 1-25, 1995. 
Friston KJ, Holmes A, Poline J-B, Price CJ, And Frith CD. Detecting activations in PET and fMRI, levels of inference and power. Neuroimage 40: 223-235, 1996.

Geyer S, Ledberg A, Schleicher A, Kinomura S, Schormann T, Burgel U, Klingberg T, Larsson J, Zilles K, And Roland PE. Two different areas within the primary motor cortex of man. Nature 382: 805-807, 1996.

Geyer S, Schleicher A, And Zilles K. Areas 3a, 3b, and 1 of human primary somatosensory cortex. 1. Microstructural organization and interindividual variability. Neuroimage 10: 63-83, 1999.

Grafton ST, HAZELTINE E, AND IVRY R. Functional mapping of sequence learning in normal human. J Cognit Neurosci 7: 497-510, 1995.

Hari R, Forss N, Avikainen S, Kiveskari E, Selenius S, and Rizzolatti G. Activation of primary motor cortex during action observation: a neuromagnetic study. Proc Natl Acad Sci USA 95: 15061-15065, 1998.

Hsiao SS, O'Shaughnessy DM, AND Johnson KO. Effects of selective attention on spatial form processing in monkey primary and secondary somatosensory cortex. J Neurophysiol 70: 444-447, 1993.

IRIKI A, TANAKA M, AND IwAmURA Y. Attention-induced neuronal activity in the monkey somatosensory cortex revealed by pupillometrics. Neurosci Res 25: 173-181, 1996.

LEYTON AS AND SHERRINGTON CS. Observations on the excitable cortex of the chimpanzee, orang-utan and gorilla. $Q J$ Exp Physiol 11: 135-222, 1917.

Lurito JT, Georgakopoulos T, AND Georgopoulos AP. Cognitive spatialmotor processes. 7. The making of movements at an angle from a stimulus direction at the single cell and population levels. Exp Brain Res 87: 562$580,1991$.

Mahalanobis PC, Majumda DN, And Rao CR. Anthropometric survey of the united provinces. A statistical study. Sankhya 9: 89-324, 1949.

MALONEK D AND GRINVALD A. Interaction between electrical activity and cortical microstimulation revealed by imaging spectroscapy: implications for functional brain mapping. Science 272: 551-554, 1996.

MERKER B. Silver staining of cell bodies by means of physical development. J Neurosci Methods 9: 235-241, 1983.

OLDFIELD RC. The assessment and analysis of handedness: the Edinburgh inventory. Neuropsychologia 9: 97-113, 1971.

Passingham RE. Attention to action. Philos Trans R Soc Lond B Biol Sci 351: 1473-1479, 1996.

Pellizzer G, Sargent P, and Georgopoulos AP. Motor cortical activity in a context-recall task. Science 269: 702-705, 1995.

Penfield W and Rasmussen T. The Cerebral Cortex of Man. New York: Macmillan, 1950.

PRICE CJ AND FRISTON KJ. Cognitive conjunction: a new experimental design for fMRI. Neurolmage 5: 261-270, 1997.

Roland PE, Graufelds CJ, WÅHLin J, Ingelman L, ANDERSSON M, LEDBERG A, Pedersen J, Åkerman S, Dabringhaus A, and Zilles K. Human brain atlas: for high-resolution functional and anatomical mapping. Hum Brain Mapp 1: 173-184, 1994.

Roland PE AND ZiLles K. Brain atlases-a new research tool. Trends Neurosci 17: 458-467, 1994.

Schleicher A, Amunts K, Geyer S, Kowalski T, Schormann T, PalomeroGallagher N, AND ZILles K. A stereological approach to human cortical architecture: identification and delineation of cortical areas. J Chem Neuroanat 20: 31-47, 2000.

Schleicher A, Amunts K, Geyer S, Morosan P, and Zilles K. Observerindependent method for microstructural parcellation of cerebral cortex: a quantitative approach to cytoarchitectonics. Neuroimage 9: 165-177, 1999.

SCHLEICHER A AND ZiLles K. A quantitative approach to cytoarchitectonics: analysis of structural inhomogeneities in nervous tissue using an image analyser. J Microsc 157: 367-381, 1990.

Schnitzler A, Salenius S, Salmelin R, Jousmaki V, and Hari R. Involvement of primary motor cortex in motor imagery; a neuromagnetic study. Neuroimage 6: 201-208, 1997.

Schormann T, Henn S, And Zilles K. A new approach to fast elastic alignment with application to human brains. Lect Notes Comput Sci 1131: 437-442, 1996.

SCHORMANN T AND ZILLES K. Three-dimensional linear and nonlinear transformations: an integration of light microscopical and MRI data. Hum Brain Mapp 6: 339-347, 1998.

SHEN L AND ALEXANDER GE. Neural correlates of a spatial sensory-to-motor transformation in primary motor cortex. J Neurophysiol 77: 1171-1194, 1997.

Shulman GL, Corbetta M, Buckner RL, Raichle ME, Fiez JA, Miezin FM, And Petersen SE. Top-down modulation of early sensory cortex. Cereb Cortex 7: 193-206, 1997.

Smyrnis N, TAira M, Ashe J, And Georgopoulos AP. Motor cortical activity in memorized delay task. Exp Brain Res 92: 139-151, 1992.

Steinmetz PN, Roy A, Fitzgerald PJ, Hsiao SS, Johnson KO, and Niebur E. Attention modulates synchronized neuronal firing in primate somatosensory cortex. Nature 404: 131-133, 2000.

STEPNIEWSKa I, PREUSS TM, AND KAAS JH. Architectonics, somatotopic organization, and ipsilateral cortical connections of the primary motor area (M1) of owl monkeys. J Comp Neurol 330: 238-271, 1993.

StePniEwsKa I, Preuss TM, and KaAs JH. Thalamic connections of the primary motor cortex (M1) of owl monkeys. J Comp Neurol 349: 558-582, 1994.

Talairach J And Turnoux P. Co-Planar Stereotactic Atlas of the Human Brain. Stuttgart, Germany: Thieme Medical Publishers, 1988.

Woldorf MG, Gallen CC, Hampson SA, Hillyard SA, Pantev C, Sobel D, AND BLOOM FE. Modulation of early sensory processing in human auditory cortex during auditory selective attention. Proc Natl Acad Sci USA 15: 8722-8726, 1993. 